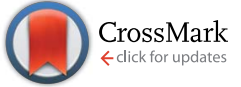

Cite this: RSC Adv., 2017, 7, 13561

Received 8th November 2016 Accepted 23rd February 2017

DOI: $10.1039 / c 6 r a 26470 k$

rsc.li/rsc-advances

\title{
The fluorescence quenching phenomenon in newly synthesized blue fluorescence protein molecule caused by anchoring group substitution: a DFT and TD-DFT study $\dagger$
}

\begin{abstract}
Chi Ma, Yufang Liu, ${ }^{*}$ Chaozheng Li and Yonggang Yang
The newly synthesized blue fluorescence protein (BFP) molecule combined with its derivatives were fully investigated using DFT and TD-DFT methods. The frontier molecular orbitals and NBO charges indicate that the intramolecular benzene ring in the BFP molecule can inhibit the charge redistribution after photo-excitation effectively. The substitution of malonic and malononitrile groups can create the fluorescence quenching phenomenon, which may be caused by their participation in the excited state charge redistribution and act as important electron-donating groups. Also, these substitutions can enhance the configuration stability in the $S_{1}$ state and hinder the formation of a metastable structure. The malononitrile group substitution can significantly decrease the energy barrier in the $S_{1}$ state and promote proton transfer
\end{abstract}

\section{Introduction}

Excited state proton transfer (ESPT) chromophores play critical roles in many physical and biological systems, such as fluorescence sensors, ${ }^{1}$ laser dyes, ${ }^{2}$ UV filters, ${ }^{3}$ molecular switches ${ }^{4}$ and LEDs, ${ }^{5}$ as they can create some special photo-physical and photo-chemical phenomenon and undergo drastic structural reorganization and charge redistribution after photo-excitation. Since the excited-state proton transfer phenomenon was first observed in the experiment with methyl salicylate by Wille and co-workers, ${ }^{6}$ a vast number of scientists have directed their attention to clarify the specific excited state proton transfer mechanism. ${ }^{7-11}$ Several decades ago, people began to realize the fundamental importance of weak interaction in the excited state proton transfer process, especially the hydrogen bond ${ }^{12}$ with its directional character. Han et al. ${ }^{13}$ proposed that the intra- and intermolecular hydrogen bond strengthening after photoexcitation can facilitate the proton transfer effectively. As one of the most widely investigated excited state intermolecular proton transfer (ESIPT) biological chromophores, fluorescent proteins ${ }^{14,15}$ have drawn great attention due to their unique properties and numerous applications in molecular biology. For instance, the green fluorescence protein (GFP) ${ }^{16}$ chromophores have been used as powerful tools in bio-imaging technology due to their high fluorescence quantum yields. However, the overall

College of Physics and Material Science, Henan Normal University, Xinxiang 453007, China. E-mail: yf-liu@htu.cn; Fax: +86 373 3329297; Tel: +86 3733329297

$\dagger$ Electronic supplementary information (ESI) available. See DOI: $10.1039 / \mathrm{c} 6 \mathrm{ra} 26470 \mathrm{k}$ performance, such as the brightness and stability, still need to be improved. Compared with the relative comprehensively investigated GFP chromophore, the previous studies about blue fluorescence proteins (BFP) ${ }^{17}$ chromophores were mainly focused on the spectroscopic properties, relative little is known about the specific reaction dynamic process and mechanism. Recently, Fang and co-workers ${ }^{18}$ have synthesized a new blue fluorescent protein chromophore analogue 2-BFP $((4 Z)-4-[(1 H-$ imidazol-2-yl)methylene]-1-methyl-2-phenyl-1 $H$-imidazol-5-(4H)one), which plays dual fluorescent emission and larger Stokes shift. In contrast with other fluorescent protein analogues, the nitrogen atoms were acted as both donor and acceptor moieties $(\mathrm{N}-\mathrm{H} \cdots \mathrm{N})$ in the 2-BFP molecule, it also contains greatly enhanced quantum yields and much slower proton-transfer rates. The previous investigation on ESPT structures, ${ }^{19,20}$ such as hydroxyphenylbenzoxazole $(\mathrm{HBO})^{21}$ and hydroxyphenylimidazopyridine (HPIP), ${ }^{22}$ are typical $\mathrm{O}-\mathrm{H} \cdots \mathrm{N}$ systems with the intramolecular hydrogen bond forming a six-membered ring. Therefore, the investigation on the newly synthesized 2-BFP chromophore with $\mathrm{N}-\mathrm{H} \cdots \mathrm{N}$ hydrogen bond can propose further understanding about the underlying photophysical and photochemical mechanism of natural fluorescence proteins. In the present work, the newly synthesized blue fluorescent protein was set as the basic configuration under analyzing. The truncated BFP chromophore (T-BFP) was set as a comparison to investigate the influence of intramolecular benzene ring. And some classical anchoring groups were introduced to substitute the methyl group in the BFP chromophore to investigate the specific influence on spectral properties and excited state dynamic process. The potential energy curves were scanned with a series of fixed bond lengths 
and dihedral angles. The electronic spectra, frontier molecular orbitals and NBO charges were also been presented.

\section{Computational details}

In the present work, all the geometry configurations were fully optimized based on DFT ${ }^{23,24}$ method in ground state and TDDFT $^{25,26}$ method in excited state by Gaussian 09 (ref. 27) program package. The popular Becke's three-parameter hybrid exchange functional with Lee-Yang-Parr gradient-corrected correlation (B3LYP $)^{28-31}$ as well as the standard $6-31++\mathrm{G}(\mathrm{d}, \mathrm{p})$ level were performed in both ground and excited states. For the previous investigations ${ }^{20,32-35}$ have proved the applicability on this kind of configurations. In order to reduce the influence of external environment, all the optimizations were performed in the adiabatic environment. Benzene was chosen as the solvent throughout based on the integral equation formalism variant of the polarizable continuum model (IEFPCM). ${ }^{36-38}$ The newly synthesized blue fluorescent protein chromophore and its anchoring group substituted derivatives were fully optimized in both $S_{0}$ and $S_{1}$ states without any constraint of bonds, angles and dihedral angles. The vertical excitation energies were calculated by TDDFT method with IEFPCM based on the ground state optimized structures. The theoretical calculation provided six low-lying absorbing transitions and all the local minima geometries were confirmed without any imaginary modes in the vibrational analysis. Meanwhile, the potential energy curves were scanned in both $\mathrm{S}_{0}$ and $\mathrm{S}_{1}$ states with a series of fixed $\mathrm{N}-\mathrm{H}$ bonds and dihedral angles in benzene with adiabatic condition. Harmonic vibrational frequencies in $S_{0}$ and $S_{1}$ states were determined by diagonalization of the Hessian. ${ }^{39}$ The excitedstate Hessian was obtained by numerical differentiation of the analytical gradients using central differences and default displacements of 0.02 bohr. $^{40}$

\section{Results and discussion}

\subsection{The influence of intramolecular benzene ring}

In order to clarify the specific influence of intramolecular benzene ring of BFP chromophore, the truncated BFP (T-BFP) and BFP molecule were fully optimized using the B3LYP function with $6-31++G(d, p)$ basis set in vacuum (shown in Fig. 1). All the optimized structures mentioned above were confirmed with the minima energy and real frequencies. Some of the notable atoms involved in the analysis were marked and shown in Fig. 1. The bond lengths and angles in the hydrogen bond were presented in Table 1.

As is shown in Table 1, the $\mathrm{N}-\mathrm{H}$ bond in BFP chromophore slightly lengthens from $1.017 \AA\left(\mathrm{S}_{0}\right)$ to $1.020 \AA\left(\mathrm{S}_{1}\right)$. The intramolecular hydrogen bond $\mathrm{H} \cdots \mathrm{N}$ shortens from $2.161 \AA\left(\mathrm{S}_{0}\right)$ to $2.083 \AA\left(\mathrm{S}_{1}\right)$ combined with the $\delta_{(\mathrm{N}-\mathrm{H} \cdots \mathrm{N})}$ enlarge from $123.11^{\circ}$ $\left(\mathrm{S}_{0}\right)$ to $125.10^{\circ}\left(\mathrm{S}_{1}\right)$ after photo-excitation. The similar configuration data and variation tendency can be found in the T-BFP molecule. Therefore, it can be concluded that the intramolecular hydrogen bonds were strengthened after photoexcitation in both BFP and T-BFP molecules. As for the proton transfer isomer, the hydrogen bond in BFP-T changes from

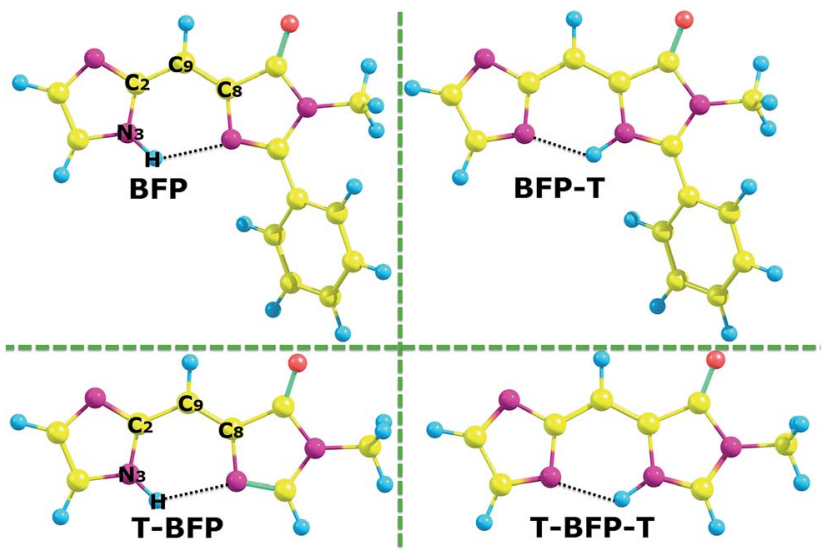

Fig. 1 The optimized structures of BFP and T-BFP combined with their proton transferred configurations (-T).

$1.966 \AA\left(\mathrm{S}_{0}\right)$ to $1.891 \AA\left(\mathrm{S}_{1}\right)$ and that in T-BFP-T only changes from $1.968 \AA\left(\mathrm{S}_{0}\right)$ to $1.957 \AA\left(\mathrm{S}_{1}\right)$, which means that the reverse proton transfer is much easier happened in the BFP-T molecule (Table 2).

The first excited states optimizations were performed by TD-DFT/B3LYP/6-31++G(d,p) and confirmed with real frequency. The absorption peak for BFP chromophore from ground state to first excite state was located at $419 \mathrm{~nm}$ with the large oscillator strength of 0.7287 , while the $\mathrm{S}_{0} \rightarrow \mathrm{S}_{2}$ transition was calculated to be $343 \mathrm{~nm}$ with the oscillator strength of 0.0034 . And the photo-excitation in T-BFP chromophore was also mainly from $S_{0}$ to $S_{1}$ state with large oscillator strength of 0.7117 . Therefore, only the HOMO and LUMO orbitals were discussed in detail, which are shown in the ESI. $\dagger$ Both the BFP and T-BFP molecules clearly show $\pi$ character for the highest occupied molecular orbital (HOMO) and $\pi^{*}$ character for the lowest unoccupied molecular orbital (LUMO), with a predominant $\pi \pi^{*}$-type transition from HOMO to LUMO. Except for the extension to the benzene ring, the electron distribution in BFP and T-BFP molecules have no clear difference.

In order to give a more accurate description on the charge redistribution after photo-excitation, the NBO charges was presented in Fig. 2. In T-BFP, the negative charge located on imidazole decreases from $0.082\left(\mathrm{~S}_{0}\right)$ to $0.112\left(\mathrm{~S}_{1}\right)$ together with that increasement on 4-oxygen-imidazole from $-0.719\left(\mathrm{~S}_{0}\right)$ to $-0.757\left(\mathrm{~S}_{1}\right)$. It means that the electron mainly transfer from imidazole to 4-oxygen-imidazole after photo-excitation. In BFP, the electron located on imidazole decrease from $0.073\left(\mathrm{~S}_{0}\right)$ to $0.088\left(\mathrm{~S}_{1}\right)$, however, that on 4-oxygen-imidazole only increases from $-0.511\left(\mathrm{~S}_{0}\right)$ to $-0.529\left(\mathrm{~S}_{1}\right)$. This means that the participation of benzene ring inhibit the electron density increasement on 4-oxygen-imidazole after photo-excitation. The charge redistribution on the atoms involved in the intramolecular hydrogen bond can be considered as a reliable evidence to study the excited state hydrogen bond. In T-BFP, the electron on $\mathrm{H}_{1}$ atom decreases from $0.477\left(\mathrm{~S}_{0}\right)$ to $0.480\left(\mathrm{~S}_{1}\right)$ together with that increasement on $\mathrm{N}_{2}$ atom from $-0.562\left(\mathrm{~S}_{0}\right)$ to $-0.583\left(\mathrm{~S}_{1}\right)$. In $\mathrm{BFP}$, the electron on $\mathrm{H}_{1}$ atom maintain 0.476 in both $\mathrm{S}_{0}$ and $\mathrm{S}_{1}$ 
Table 1 The calculated bond lengths $(\AA \AA)$, bond angles $\left(^{\circ}\right)$ and Wiberg bond index

\begin{tabular}{|c|c|c|c|c|c|c|}
\hline & & \multicolumn{2}{|l|}{$\mathrm{N}-\mathrm{H}$} & \multicolumn{2}{|l|}{$\mathrm{H} \cdots \mathrm{N}$} & \multirow[b]{2}{*}{$\delta_{(\mathrm{N}-\mathrm{H} \cdots \mathrm{N})}$} \\
\hline & & Bond length & Wiberg index & Bond length & Wiberg index & \\
\hline \multirow[t]{2}{*}{ BFP } & $\mathrm{S}_{0}$ & 1.017 & 0.7211 & 2.161 & 0.0253 & 123.11 \\
\hline & $\mathrm{S}_{1}$ & 1.020 & 0.7128 & 2.083 & 0.0333 & 125.10 \\
\hline \multirow[t]{2}{*}{ BFP-T } & $\mathrm{S}_{0}$ & 1.966 & 0.0601 & 1.031 & 0.6699 & 128.17 \\
\hline & $\mathrm{S}_{1}$ & 1.891 & 0.0619 & 1.033 & 0.6710 & 128.94 \\
\hline \multirow[t]{2}{*}{ T-BFP } & $\mathrm{S}_{0}$ & 1.017 & 0.7216 & 2.170 & 0.0243 & 122.90 \\
\hline & $\mathrm{S}_{1}$ & 1.019 & 0.7115 & 2.105 & 0.0315 & 124.54 \\
\hline \multirow[t]{2}{*}{ T-BFP-T } & $\mathrm{S}_{0}$ & 1.968 & 0.0590 & 1.030 & 0.6708 & 128.40 \\
\hline & $\mathrm{S}_{1}$ & 1.957 & 0.0719 & 1.026 & 0.6566 & 127.59 \\
\hline
\end{tabular}

Table 2 Electronic excitation energy $(\mathrm{nm})$, corresponding oscillator strengths, and the corresponding compositions for BFP and truncated BFP

\begin{tabular}{llllll}
\hline & Transition & $\lambda(\mathrm{nm})$ & $f$ & Composition & CI (\%) \\
\hline BFP & $\mathrm{S}_{0} \rightarrow \mathrm{S}_{1}$ & 419 & 0.7287 & $\mathrm{H} \rightarrow \mathrm{L}$ & $99 \%$ \\
& $\mathrm{~S}_{0} \rightarrow \mathrm{S}_{2}$ & 343 & 0.0034 & $\mathrm{H}-1 \rightarrow \mathrm{L}$ & $6 \%$ \\
T-BFP & $\mathrm{S}_{0} \rightarrow \mathrm{S}_{1}$ & 385 & 0.7117 & $\mathrm{H} \rightarrow \mathrm{L}$ & $99 \%$ \\
& $\mathrm{~S}_{0} \rightarrow \mathrm{S}_{2}$ & 342 & 0.0005 & $\mathrm{H}-1 \rightarrow \mathrm{L}$ & $5 \%$ \\
\hline
\end{tabular}

states, it only slightly increases from $-0.554\left(\mathrm{~S}_{0}\right)$ to $-0.566\left(\mathrm{~S}_{1}\right)$ on $\mathrm{N}_{2}$ atom.

For describing the influence of intramolecular benzene ring on the electronic spectra, the corresponding absorption and emission spectra of T-BFP and BFP molecules were displayed in
Fig. 3. The calculated absorption peaks of T-BFP and BFP are located at $385 \mathrm{~nm}$ and $420 \mathrm{~nm}$, respectively. And the emission peak of BFP is located at $505 \mathrm{~nm}$, which strongly redshift compared with that of T-BFP at $435 \mathrm{~nm}$. The structural reorganization caused by excited state proton transfer makes the Stokes shift enlarges from $85 \mathrm{~nm}$ to $145 \mathrm{~nm}$ in BFP, however, it only changes from $50 \mathrm{~nm}$ to $79 \mathrm{~nm}$ in T-BFP. Therefore, it is reasonable to believe that the benzene ring has an appreciable impact on the spectral property.

In order to provide an unambiguous information about ESIPT process, the potential energies were scanned with a series of constrained $\mathrm{N}-\mathrm{H}$ bond from $1.01 \AA$ to $2.21 \AA$ in step of $0.1 \AA$ in both $S_{0}$ and $S_{1}$ states (shown in Fig. 4). In ground state, the potential energy barrier in T-BFP $\left(15.40 \mathrm{kcal} \mathrm{mol}^{-1}\right)$ is a little higher than that in BFP $\left(14.62 \mathrm{kcal} \mathrm{mol}^{-1}\right)$. After photoexcitation, the energy barriers are 10.28 and $9.08 \mathrm{kcal} \mathrm{mol}^{-1}$
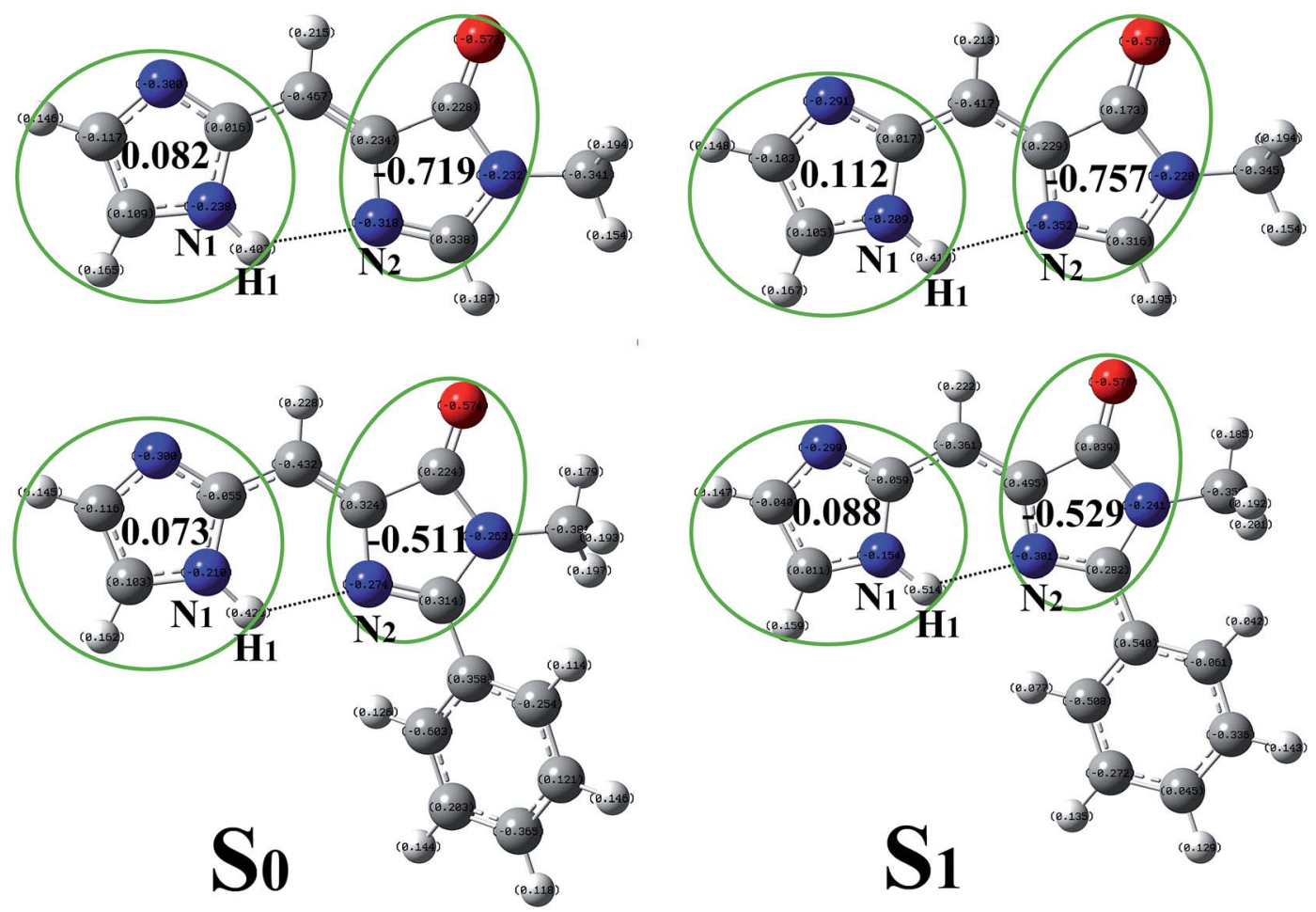

Fig. 2 The NBO charges of T-BFP and BFP molecules. 


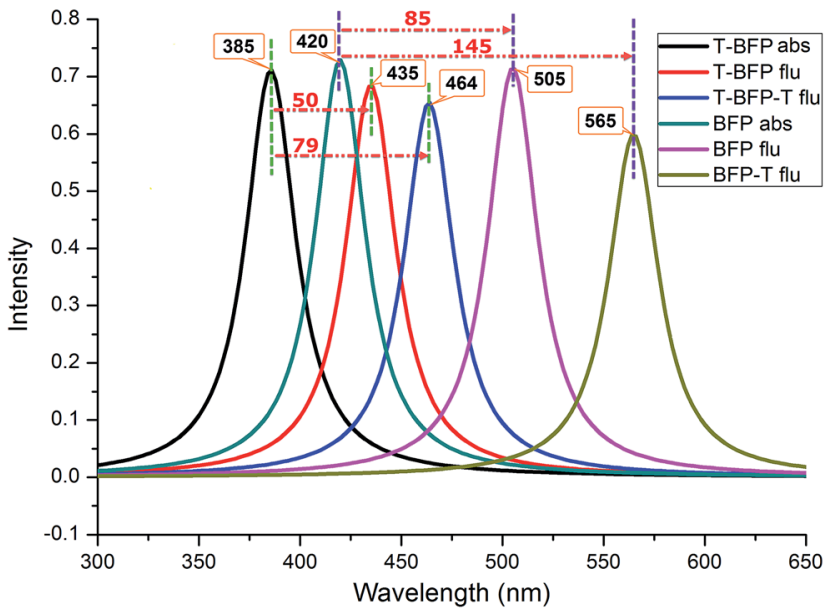

Fig. 3 The absorption (abs) and fluorescence (flu) spectra of the molecules.

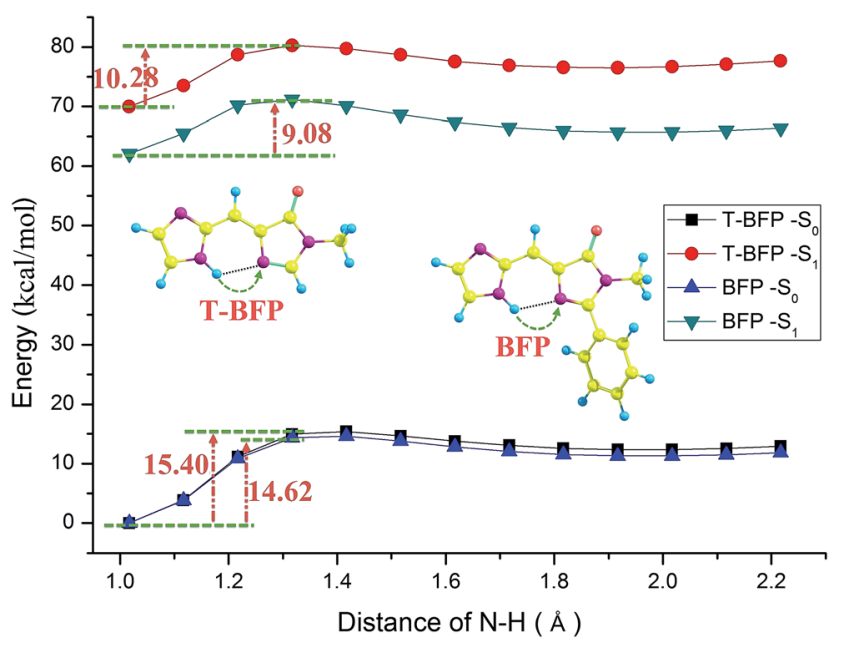

Fig. 4 The constructed potential energy curves with constrained $\mathrm{N}-\mathrm{H}$ bond lengths.

in T-BFP and BFP, respectively. In brief, the energy barriers are relative lower in BFP molecule in both ground and first excited state, which means the intramolecular benzene ring may be conducive to proton transfer. It also can be noted that the overall energy distribution of BFP in $\mathrm{S}_{1}$ state is visible lower than that of T-BFP, indicating that the BFP molecule is more stable in first excited state.

As for the torsional stability, the potential energies were scanned with a series of constrained dihedral angles $\left(\mathrm{N}_{3}-\mathrm{C}_{2}-\right.$ $\mathrm{C}_{9}-\mathrm{C}_{8}$ ) from $0^{\circ}$ to $180^{\circ}$ in step of $10^{\circ}$ in both $S_{0}$ and $S_{1}$ states (Fig. 5). In ground state, the energy barriers were calculated to be 14.26 and $14.55 \mathrm{kcal} \mathrm{mol}^{-1}$ in BFP and T-BFP, respectively. It is surprising to find that a metastable configuration was formed in both BFP and T-BFP molecules with the imidazole ring twist about $90^{\circ}$ after photo-excitation. In BFP, it needs to conquer $12.94 \mathrm{kcal} \mathrm{mol}^{-1}$ to form the metastable configuration. And there is only $8.95 \mathrm{kcal} \mathrm{mol}^{-1}$ separate the original T-BFP molecule and its metastable structure. The intramolecular benzene ring can enhance the configuration stability in first excited state (Fig. 6).

\subsection{The influence of anchoring group substitution}

Some classical anchoring groups with weak electron pulling strength (-C, -AC, -MC) and strong electron pulling strength (-CC, -MN) were employed to substitute the methyl group in BFP chromophore. All the BFP derivatives were fully optimized in both $S_{0}$ and $S_{1}$ states with Gaussian 09 program. The bond lengths and angles involved in the hydrogen bond only have insignificant difference in both $S_{0}$ and $S_{1}$ states after substituted by several anchoring groups. The reasonable deducing can be presented that the anchoring groups only have a trifling influence on the hydrogen bond. As for the whole configuration, the anchoring group become vertical to the trunk in BFP-MC and BFP-MN in $\mathrm{S}_{1}$ state. The significant configuration variations may provide some unique properties in excited state.

As is shown Fig. 7, the absorption peaks of these derivatives are spanning from $420 \mathrm{~nm}$ to $428 \mathrm{~nm}$, which means the absorption property is not sensitive to anchoring groups. Due to the neglectful oscillator strength with 0.0004 (BFP-MC) and 0.0012 (BFP-MN), only three emission spectra (BFP-AC, -C, -CC) are presented in this figure. The fluorescence quenching phenomenon in the newly synthesized blue fluorescence protein induced by anchoring group substitution was firstly proposed in this work. In order to make clear the immanent cause of the fluorescence quenching phenomenon, the NBO charges were presented to be rigorously studied (Fig. 8).

As is shown in Fig. $\mathrm{S} 2, \uparrow$ the electron density on $\mathrm{N}_{1}, \mathrm{H}_{1}$ and $\mathrm{N}_{2}$ atoms have no significantly change after photoexcitation, which is according with the insignificant change of hydrogen bond discussed above. The substituted anchoring group was set as part $\mathrm{c}$ to discuss the excited state charge redistribution. In BFP$\mathrm{C}$ molecule, the electron density on imidazole decreases from $0.136\left(\mathrm{~S}_{0}\right)$ to $0.156\left(\mathrm{~S}_{1}\right)$ and that on 4-oxygen-imidazole increases from $-0.542\left(\mathrm{~S}_{0}\right)$ to $-0.558\left(\mathrm{~S}_{1}\right)$. However, the electron density on carboxylic acid only has a tiny decrease about 0.007 after photo-excitation. The similar changes also be found in BFP-CC and BFP-AC molecules. Therefore, it can be reasonably concluded that the electron was mainly transfer from imidazole to 4-oxygen-imidazole (Fig. 9).

In BFP-MC molecule, the electron density on imidazole decreases 0.014 from $0.127\left(\mathrm{~S}_{0}\right)$ to $0.141\left(\mathrm{~S}_{1}\right)$ and that on 4 oxygen-imidazole increases 0.065 from $-0.477\left(\mathrm{~S}_{0}\right)$ to -0.542 $\left(\mathrm{S}_{1}\right)$. The electron on the malonic acid clearly decreases 0.1 from $0.125\left(\mathrm{~S}_{0}\right)$ to $0.225\left(\mathrm{~S}_{1}\right)$, which means the anchoring group is considered to be an important electron-donating group. As for BFP-MN molecule, it also found the diminution on the malononitrile acid is 0.126 from $0.086\left(\mathrm{~S}_{0}\right)$ to $0.212\left(\mathrm{~S}_{1}\right)$. In brief, the unusual fluorescence properties of BFP-MC and BFP-MN can be explained by the participation of anchoring group in charge redistribution.

The potential energy curves were scanned with a series of fixed $\mathrm{N}-\mathrm{H}$ bonds in both $\mathrm{S}_{0}$ and $\mathrm{S}_{1}$ states to clarify the influence of anchoring groups on intramolecular proton transfer. Some incidental information are listed in the ESI and shown 

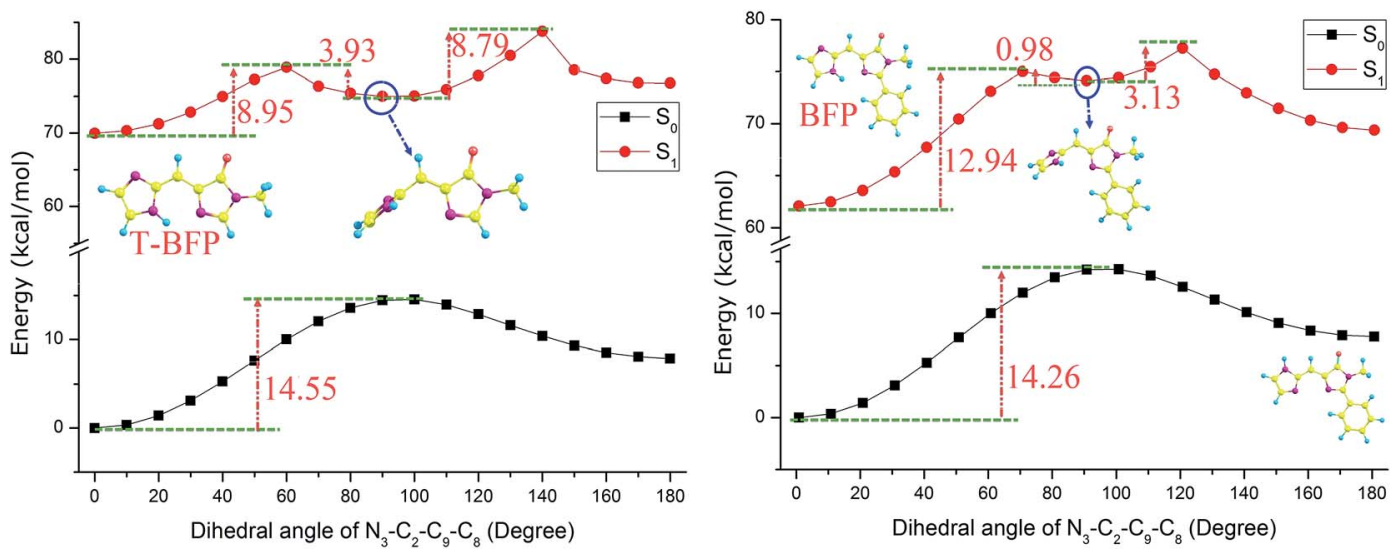

Fig. 5 The constructed potential energy curves of T-BFP and BFP molecules with constrained dihedral angles.

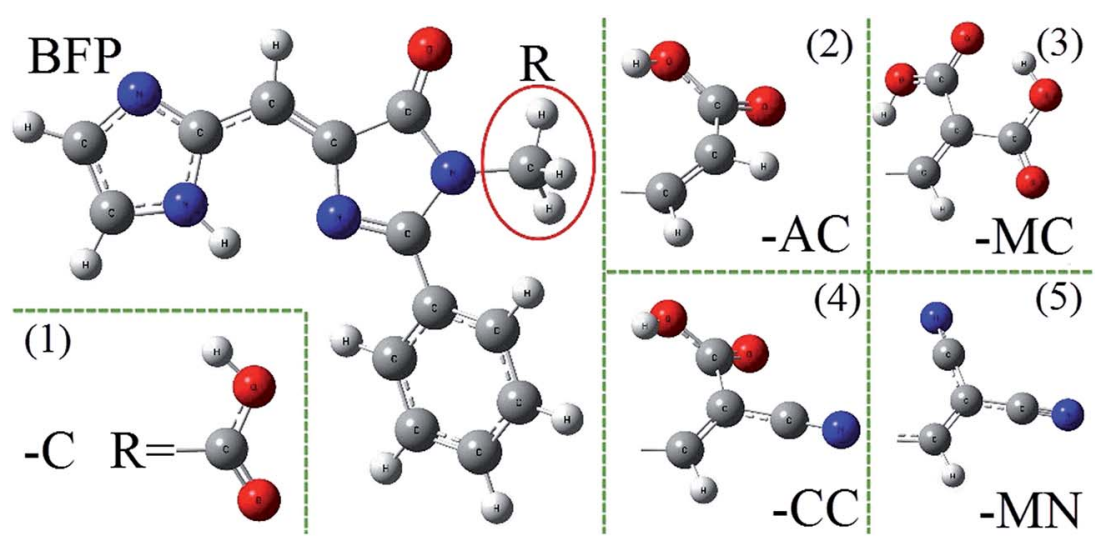

Fig. 6 The structures of anchoring groups: (1) carboxylic acid, (2) acrylic acid, (3) malonic acid, (4) cyanoacrylic acid and (5) malononitrile acid.

as Fig. S3 and Table S1.† In ground state, the energy barriers of the substitution derivatives have distinct increasement compared with that of the original BFP molecule, which means the anchoring groups can hinder the proton transfer in ground state. The result also reveal that the proton transfer

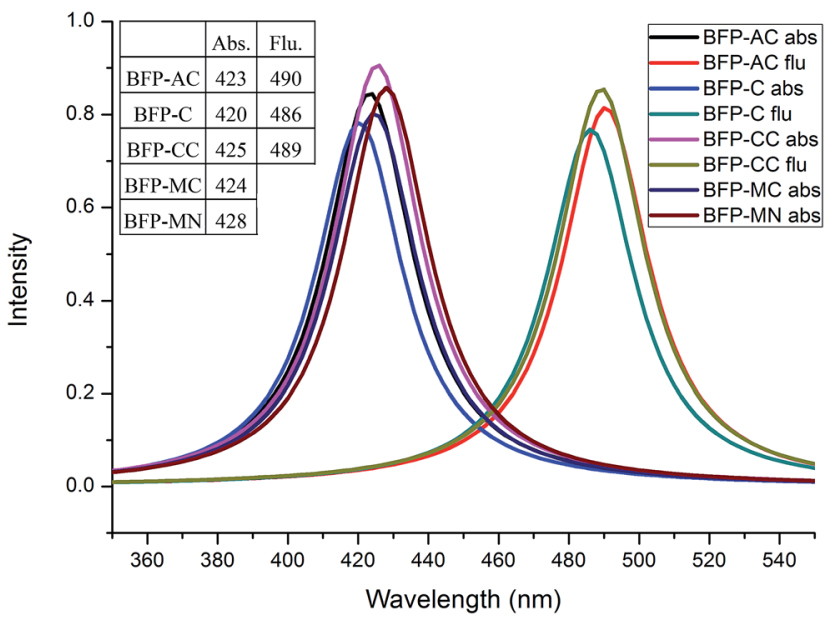

Fig. 7 The absorption and emission spectra of BFP derivatives. probability of BFP substitution derivatives in ground state follow the sequence BFP $<$ BFP-C $<$ BFP-AC $<$ BFP-CC $<$ BFP-MC $<$ BFP-MN. After photo-excitation, the substitution derivatives, except for BFP-MN molecule, also show proton transfer restrain phenomenon with the compared increasement of potential energy barrier. However, it is clearly shown that the energy barrier of BFP-MN molecule distinctly decreased to $1.17 \mathrm{kcal} \mathrm{mol}^{-1}$ in first excited state. Hence, the substitution of malononitrile group can significantly promote the proton transfer after photo-excitation. Also, a similar sequence of proton transfer probability in first excited state can be presented, which is BFP-MN $<$ BFP $<$ BFP-MC $<$ BFP-C $<$ BFP-AC $<$ BFP-CC.

As is shown in Fig. 10, the BFP substitution derivatives were scanned with a series of fixed dihedral angles in both $S_{0}$ and $S_{1}$ states. Compared with the original BFP molecule, the energy barriers of the derivatives in ground state are increased after being substituted by anchoring groups. Among these derivatives, the BFP-CC molecule has the most obvious enhancement, which increase about $1.85 \mathrm{kcal} \mathrm{mol}^{-1}$. It can be deduced that the anchoring group will hinder the configuration twist and maintain the molecule in the same plane. As discussed above, in the $\mathrm{S}_{1}$ state, the imidazole ring can twist about $90^{\circ}$ to produce the 


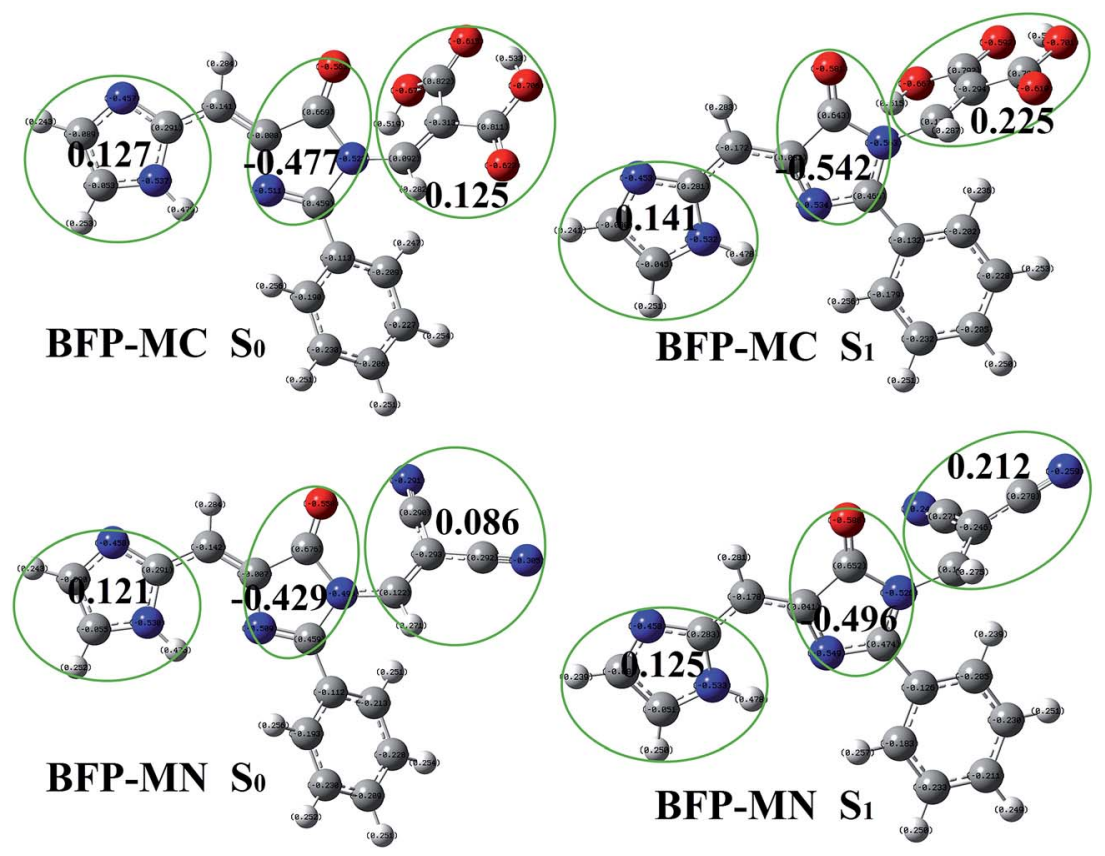

Fig. 8 The NBO charges of BFP-MC and BFP-MN in both $S_{0}$ and $S_{1}$ states.

metastable configuration. It can be noted that the BFP-C, BFP-AC and BFP-CC molecules are much easier transferring to the metastable state, for their potential energies are visibly decreased compared with the original BFP molecule in $\mathrm{S}_{1}$ state, which means their stability are weakened in excited state. However, it is surprised to note that the potential energies of BFP-MC and BFP-
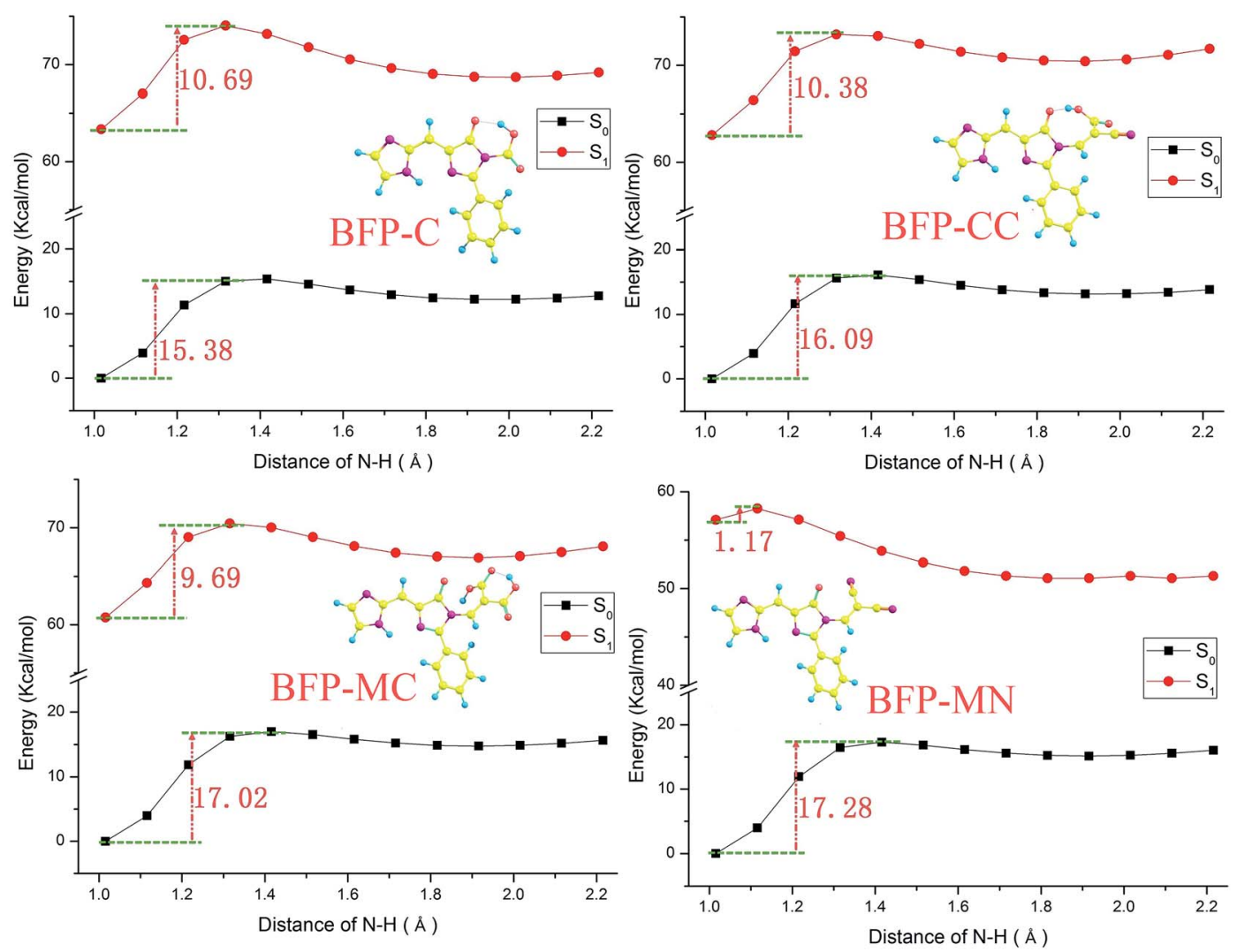

Fig. 9 The potential energy curves with a series of fixed $\mathrm{N}-\mathrm{H}$ bond lengths. 

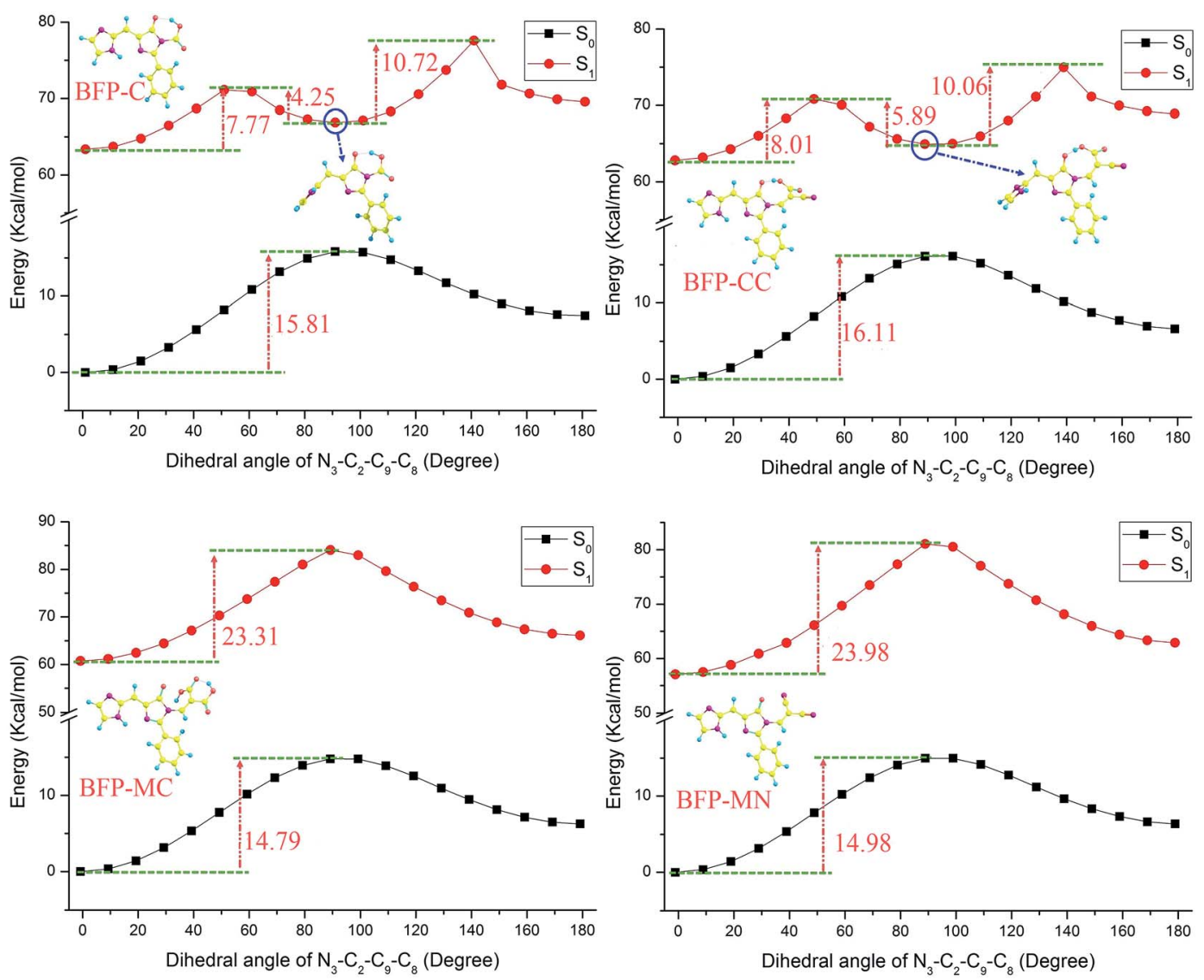

Fig. 10 The potential energy curves with a series of fixed dihedral angles.

MN molecules sharply raised to 23.31 and $23.98 \mathrm{kcal} \mathrm{mol}^{-1}$, respectively. And the metastable configuration are not existed in these molecules. In brief, the participation of anchoring groups enhance the ground state stability. Particularly, the malonic and malononitrile group significantly enhance the configuration stability in first excited state and hinder the formation of metastable configuration in excited state.

\section{Conclusion}

In the present work, the BFP molecule was truncated to investigate the effect of intramolecular benzene ring. The bond and angle analysis indicates that the benzene ring only has a trifling influence on the intramolecular hydrogen bond. Based on the frontier molecular orbitals and NBO charges, it found that the intramolecular benzene ring can inhibits the charge redistribution after photo-excitation. We also found the absorption peak redshift from $385 \mathrm{~nm}$ (T-BFP) to $420 \mathrm{~nm}$ (BFP) and the Stokes shift enlarge from $79 \mathrm{~nm}$ (T-BFP) to $145 \mathrm{~nm}$ (BFP), which means the benzene ring can influence the electronic spectra of BFP molecule. The potential energy curves of BFP and T-BFP molecule were scanned with a series of fixed hydrogen bonds and dihedral angles in both ground and first excited states. The clear comparison indicates that the benzene ring can lower the energy barrier in first excited state and promote the intramolecular proton transfer. It also help enhancing the configuration stability and maintain the molecule in the same plane.

Some classical anchoring groups, such as carboxylic acid, acrylic acid, malonic acid, cyanoacrylic acid and malononitrile acid, were introduced to substitute the methyl group in the BFP molecule. It is found that these substitution groups only have a trifling influence on the intramolecular hydrogen bond. However, the substitution of malonic and malononitrile groups can create the fluorescence quenching phenomenon. Comparisons of the NBO charges in ground and first excited states show that these two anchoring groups can participate the charge transfer and act as an important electron-donating group. The malononitrile group substitution can lower the energy barrier significantly in first excited state and promote the intramolecular proton transfer. The malonic and malononitrile groups can enhance the stability in first excited state and hinder the formation of metastable configuration.

\section{Acknowledgements}

This work is supported by National Natural Science Foundation of China (Grant no. 11274096) and supported by Program for Innovative Research Team (in Science and Technology) in University of Henan Province (Grant no. 13IRTSTHN016), Supported by The High Performance Computing Center of Henan Normal University. 


\section{References}

1 Y. Wu, X. Peng, J. Fan, S. Gao, M. Tian, J. Zhao and S. Sun, Fluorescence sensing of anions based on inhibition of excited-state intramolecular proton transfer, J. Org. Chem., 2007, 72, 62-70.

2 J. H. Richardson, L. L. Steinmetz, S. B. Deutscher, W. A. Bookless and W. L. Schmelzinger, Measurement of Fluorescence Lifetimes of Coumarin Laser Dyes with a Mode-Locked Krypton Ion Laser, Z. Naturforsch., A: Phys. Sci., 2014, 33, 1592.

3 D. L. Giokas, A. Salvador and A. Chisvert, UV filters: From sunscreens to human body and the environment, TrAC, Trends Anal. Chem., 2007, 26, 360-374.

4 F. M. Raymo, Digital Processing and Communication with Molecular Switches, Adv. Mater., 2002, 14, 401-414.

5 F. Steuber, J. Staudigel, M. Stössel, J. Simmerer, A. Winnacker, H. Spreitzer, F. Weissörtel and J. Salbeck, White Light Emission from Organic LEDs Utilizing Spiro Compounds with High-Temperature Stability, Adv. Mater., 2000, 12, 130-133.

6 D.-C. E. Wille and P. D. W. Lüttke, $4,4,4^{\prime}, 4^{\prime}$-Tetramethyl$\Delta 2,2^{\prime}$-bipyrrolidine-3,3'-dione, a Compound having the Basic Chromophore System of Indigo, Angew. Chem., Int. Ed., 1971, 10, 803-804.

7 D. Yang, F. Zhao, R. Zheng, Y. Wang and J. Lv, A detailed theoretical investigation on the excited-state intramolecular proton-transfer mechanism of 3-BTHPB chemosensor, Theor. Chem. Acc., 2015, 134(5), 62.

8 H. Li, Y. Yang, D. Yang, Y. Liu and J. Sun, TDDFT study on the excited-state hydrogen bonding of $N$-(2-hydroxyethyl)-1,8naphthalimide and $N$-(3-hydroxyethyl)-1,8-naphthalimide in methanol solution, J. Phys. Org. Chem., 2014, 27, 170-176.

9 J. Catalán, J. C. D. Valle, F. Fabero and N. A. Garcia, The influence of molecular conformation on the stability of ultraviolet stabilizers toward direct and dye-sensitized photoirradiation: the case of 2-(2'-hydroxy-5'-methyphenyl) benzotriazole (tin p), Photochem. Photobiol., 1995, 61(2), 118123.

10 Y. Kubo, S. Y. Maeda, S. Tokita and M. Kubo, Colorimetric chiral recognition by a molecular sensor, Nature, 1996, 382, 522-524.

$11 \mathrm{~T}$. He and Y. Songjie, Recent progresses on diarylethene based photochromic switches, Chem. Soc. Rev., 2004, 33, 85-97.

12 H. Li, Y. Liu, Y. Yang, D. Yang and J. Sun, Excited-state intramolecular hydrogen bonding of compounds based on 2-(2-hydroxyphenyl)-1,3-benzoxazole in solution: A TDDFT study, Spectrochim. Acta, Part A, 2014, 133, 818-824.

13 G. J. Zhao and K. L. Han, Site-specific solvation of the photoexcited protochlorophyllide a in methanol: formation of the hydrogen-bonded intermediate state induced by hydrogen-bond strengthening, Biophys. J., 2008, 94, 38-46.

14 Y. Mochizuki, H. Nakanishi, Y. Kodera, S. Ito, Y. Yamamura, T. Kato, K. Hibi, S. Akiyama, A. Nakao and M. Tatematsu, TNF-alpha promotes progression of peritoneal metastasis as demonstrated using a green fluorescence protein (GFP)tagged human gastric cancer cell line, Clin. Exp. Metastasis, 2004, 21, 39-47.

15 W. Y. Huang, J. Aramburu, P. S. Douglas and S. Izumo, Transgenic expression of green fluorescence protein can cause dilated cardiomyopathy, Nat. Med., 2000, 6, 482-483.

16 A. Tomura, K. Goto, H. Morinaga, M. Nomura, T. Okabe, T. Yanase, R. Takayanagi and H. Nawata, The subnuclear three-dimensional image analysis of androgen receptor fused to green fluorescence protein, J. Biol. Chem., 2001, 276, 28395-28401.

17 A. E. Jablonski, R. B. Vegh, J. C. Hsiang, B. Bommarius, Y. C. Chen, K. M. Solntsev, A. S. Bommarius, L. M. Tolbert and R. M. Dickson, Optically modulatable blue fluorescent proteins, J. Am. Chem. Soc., 2013, 135, 16410-16417.

18 X. Fang, Y. Wang, D. Wang, G. Zhao, W. Zhang, A. Ren, H. Wang, J. Xu, B. R. Gao and W. Yang, Synthesized Blue Fluorescent Protein Analogue with Tunable Colors from Excited-State Intramolecular Proton Transfer through an N-H $\cdots \mathrm{N}$ Hydrogen Bond, J. Phys. Chem. Lett., 2014, 5, 92-98.

19 C. Ma, Y. Yang, C. Li and Y. Liu, TD-DFT Study of the Double Excited-State Intramolecular Proton Transfer Mechanism of 1,3-Bis(2-pyridylimino)-4,7-dihydroxyisoindole, J. Phys. Chem. A, 2015, 119, 12686-12692.

20 J. Zhao, H. Yao, J. Liu and M. R. Hoffmann, New excited-state proton transfer mechanisms for 1,8-dihydroxydibenzo[ $a, h]$ phenazine, J. Phys. Chem. A, 2015, 119, 681-688.

21 H. Roohi, F. Hejazi, N. Mohtamedifar and M. Jahantab, Excited state intramolecular proton transfer (ESIPT) in 2(2'-hydroxyphenyl)benzoxazole and its naphthalene-fused analogs: A TD-DFT quantum chemical study, Spectrochim. Acta, Part A, 2014, 118, 228-238.

22 A. Brenlla, M. Veiga, M. C. Rodríguez, M. Mosquera and F. Rodríguez-Prieto, Fluorescence of methylated derivatives of hydroxyphenylimidazopyridine. Resolution of strongly overlapping spectra and a new ESIPT dye showing very efficient radiationless deactivation, Photochem. Photobiol. Sci., 2011, 10, 1622-1636.

23 R. G. Parr, Density functional theory, Annu. Rev. Phys. Chem., 1983, 34, 631-656.

24 E. K. Gross and R. M. Dreizler, Density functional theory, Springer Science \& Business Media, 2013.

25 E. Runge and E. K. Gross, Density-functional theory for timedependent systems, Phys. Rev. Lett., 1984, 52, 997.

26 M. Marques, Time-dependent density functional theory, Springer Science \& Business Media, 2006.

27 M. J. Frisch, G. W. Trucks, H. B. Schlegel, G. E. Scuseria, M. A. Robb, J. R. Cheeseman, G. Scalmani, V. Barone, B. Mennucci, G. A. Petersson, H. Nakatsuji, M. Caricato, X. Li, H. P. Hrat-chian, A. F. Izmaylov, J. Bloino, G. Zheng, J. L. Sonnenberg, M. Hada, M. Ehara, K. Toyota, R. Fukuda, J. Hasegawa, M. Ishida, T. Nakajima, Y. Honda, O. Kitao, H. Nakai, T. Vreven, J. A. Montgomery Jr, J. E. Peralta, F. Ogliaro, M. Bearpark, J. J. Heyd, E. Brothers, K. N. Kudin, V. N. Staroverov, R. Kobayashi, J. Normand, K. Raghavachari, A. Rendell, J. C. Burant, S. S. Iyengar, J. Tomasi, M. Cossi, N. Rega, J. M. Millam, 
M. Klene, J. E. Knox, J. B. Cross, V. Bakken, C. Adamo, J. Jaramillo, R. Gomperts, R. E. Stratmann, O. Yazyev, A. J. Austin, R. Cammi, C. Pomelli, J. W. Ochterski, R. L. Martin, K. Morokuma, V. G. Zakrzewski, G. A. Voth, P. Salvador, J. J. Dan-nenberg, S. Dapprich, A. D. Daniels, O. Farkas, J. B. Foresman, J. V. Ortiz, J. Cioslowskiand and D. J. Fox, Gaussian 09, Revision A.1, Gaussian, Inc, Wallingford CT, 2009.

28 J. Paier, M. Marsman and G. Kresse, Why does the B3LYP hybrid functional fail for metals?, J. Chem. Phys., 2007, 127(2), 024103.

29 J. E. Del Bene, W. B. Person and K. Szczepaniak, Properties of Hydrogen-Bonded Complexes Obtained from the B3LYP Functional with $6-31 \mathrm{G}(\mathrm{d}, \mathrm{p})$ and $6-31+\mathrm{G}(\mathrm{d}, \mathrm{p})$ Basis Sets: Comparison with MP2/6-31+G(d,p) Results and Experimental Data, J. Phys. Chem., 1995, 99(27), 1070510707.

30 J. Tirado-Rives and W. L. Jorgensen, Performance of B3LYP density functional methods for a large set of organic molecules, J. Chem. Theory Comput., 2008, 4(2), 297-306.

31 L. A. Curtiss, K. Raghavachari, P. C. Redfern, et al., Investigation of the use of B3LYP zero-point energies and geometries in the calculation of enthalpies of formation, Chem. Phys. Lett., 1997, 270(5-6), 419-426.

32 R. Rondao, J. Seixas de Melo, M. Melo and A. Parola, ExcitedState Isomerization of Leuco Indigo, J. Phys. Chem. A, 2012, 116, 2826-2832.

33 S. Chai, G. J. Zhao, P. Song, S. Q. Yang, J. Y. Liu and K. L. Han, Reconsideration of the excited-state double proton transfer (ESDPT) in 2-aminopyridine/acid systems: role of the intermolecular hydrogen bonding in excited states, Phys. Chem. Chem. Phys., 2009, 11, 4385-4390.

34 J. Zhao, J. Chen, Y. Cui, J. Wang, L. Xia, Y. Dai, P. Song and F. Ma, A questionable excited-state double-proton transfer mechanism for 3-hydroxyisoquinoline, Phys. Chem. Chem. Phys., 2015, 17, 1142-1150.

35 Y. Shi and H. Wei, A theoretical study about three organic semiconductor based on oligothiophenes, Journal of Atomic and Molecular Sciences, 2015, 6, 161-171.

36 B. Mennucci, E. Cancès and J. Tomasi, Evaluation of Solvent Effects in Isotropic and Anisotropic Dielectrics and in Ionic Solutions with a Unified Integral Equation Method: Theoretical Bases, Computational Implementation, and Numerical Applications, J. Phys. Chem. B, 1997, 101, 1050610517.

37 E. Cances, B. Mennucci and J. Tomasi, New Integral Equation Formalism For The Polarizable Continuum Model: Theoretical Background And Applications To Isotropic And Anisotropic Dielectrics, J. Chem. Phys., 1997, 107, 3032-3041.

38 R. Cammi and J. Tomasi, Remarks on the use of the apparent surface charges (ASC) methods in solvation problems: Iterative versus matrix-inversion procedures and the renormalization of the apparent charges, J. Comput. Chem., 1995, 16, 1449-1458.

39 O. Treutler and R. Ahlrichs, Efficient molecular numerical integration schemes, J. Chem. Phys., 1995, 102, 346-354.

40 F. Furche and R. Ahlrichs, Adiabatic time-dependent density functional methods for excited state properties, J. Chem. Phys., 2002, 117, 7433-7447. 